\title{
Repair of damaged hair protein fiber by jointly using transglutaminase and keratin
}

\author{
Ziyuan $\mathrm{Li}^{\mathrm{a}, \mathrm{b}}$, Jing Xiao ${ }^{\mathrm{a}, \mathrm{b}, *}$ \\ a State Key Laboratory of Biobased Material and Green Papermaking, Qilu University of Technology, \\ Shandong Academy of Sciences, Jinan 250353 China \\ b Department of Bioengineering, Qilu University of Technology, Shandong Academy of Sciences, Jinan \\ 250353 China
}

*Corresponding author, e-mail: qlgdxj111@163.com

Received 13 Jul 2020

Accepted 19 Dec 2020

\begin{abstract}
This study focuses on the repair of damaged hair with the joint use of transglutaminase (TG enzyme) and keratin. We first prepared a TG-keratin repair solution with reasonable wool keratin and TG enzyme concentration, and used the soaking process to repair the damaged hair so as to modify protein fibers. We, then, measured the alkali solubility, fracture stress and strain, and swelling rate of the hair samples, before and after the treatment. The results showed that after treatment, samples' alkali solubility reduced from $16.14 \%$ to $4.92 \%$, fracture stress increased from $0.832 \mathrm{~N}$ to $1.668 \mathrm{~N}$, fracture strain arose from $8.81 \mathrm{~mm}$ to $10.17 \mathrm{~mm}$, and swelling rate drops from $38.34 \%$ to $2.34 \%$. Both Fourier transform infrared and X-ray diffraction spectroscopies further indicated that combined treatment with keratin and TG enzyme had significant repair effect on the damaged sites, where proteins were exposed with more amide bonds, higher crystallinity, increased percentage of crystalline area over the entire fiber area, and strengthened crosslink activities. Observation under both scanning electron microscope and atomic force microscope revealed smoother surface and fewer warped scales on the surface of hairs after combined treatment with keratin and TG enzyme. To our best knowledge, this is the first application of enzyme technology in damaged hair repair. It opens up a new research direction for green, environmentally friendly, and efficient biological hair care and will play a pivotal role in the application of various protein-based materials.
\end{abstract}

KEYWORDS: transglutaminase, keratin, hair repair, modified protein fiber

\section{INTRODUCTION}

Transglutaminase (TG enzyme) is an enzyme preparation widely used in food processing industry to catalyze the reaction of the $\varepsilon$-amino group on the lysine residue with the $\gamma$-hydroxyamide group on the glutamic acid residue to form covalent crosslinking between proteins or peptides. Recently, TG enzyme has also been applied to repair wool, silk and other protein fabrics. Both wool and hair are mainly composed of keratin and have similar lysine and glutamic acid residues contents [1], suggesting that application of TG enzyme for hair repair is highly feasible. The mechanism of hair repair by TG enzyme combined with keratin is shown in Fig. 1.

Currently, most hair care products use surfactants, which can only clean oil on the hair surface and not achieve the expected maintenance effect. Others, although exhibit a certain repair effect due to addition of a small amount of amino acids in them, have a limited repair effect because amino acids as small molecules are prone to lose. Proteins and their hydrolysates are beneficial to hair and able to increase hair moisture, softness and flexibility [2]. Among them, hydrolyzed keratin and its derivatives have been widely used in hair and skin care products due to their high smoothness, gloss, flexibility and biocompatibility [3]. As early as in 1988, Ni et al [4] used Croquat WKP to quarternarily ammonialize hydrolyzed keratin for hair care and found the product possesses a good adhesion ability before and after perm and cannot be easily washed away by shampoo. Zhang et al [5] used L-cysteine as a reducing agent to extract keratin from rabbit hair fiber and used it as the main raw material to prepare a novel keratin hair conditioner. Clara et al [6] studied the effects of two wool keratin samples on hair and assessed the changes in fiber on hair surface by measuring the contact angle; and it was proved that the product could clearly improve the moisture content and mechanical properties of hair fiber. The above keratin hair care methods are 

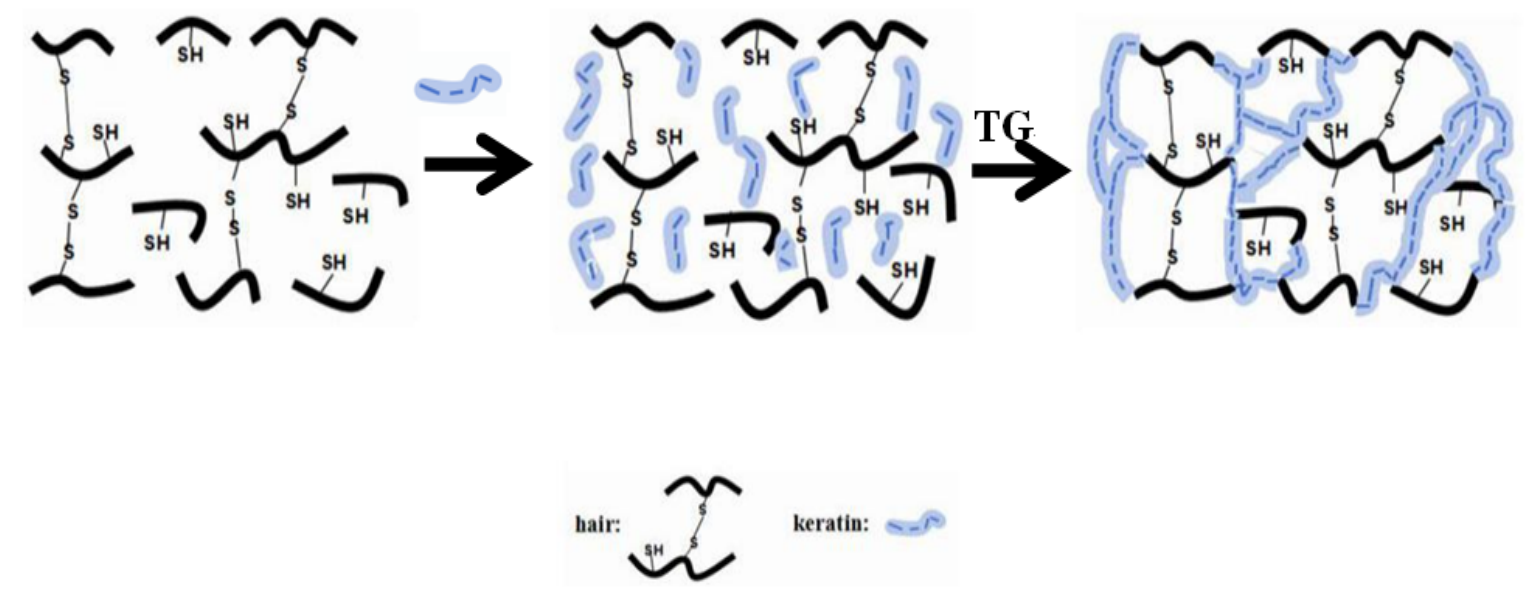

Fig. 1 Mechanism of hair repair by combination of keratin and TG enzyme.

mainly via physical adhesion to reorganize hair's broken disulfide bonds so as to change hair performance. Although this kind of hair repair has its advantages of strength improvement, moisture maintenance, and UV protection, it also has some disadvantages: (1) hair is prone to swelling due to water absorption; (2) reformation of disulfide bonds has certain randomness, and the method can only increase the formation of disulfide bond, which has limited bond energy; (3) the method is mostly based on the principle that keratin, at high concentration, could form a film. Easily peeled off film on the hair surface leads to production of massive dandruff [7-9]. In order to solve these problems, we for the first time used low concentration of keratin in combination with TG enzyme to repair damaged hairs. Compared with traditional methods, TG enzyme is a highly efficient catalyst. On the basis of forming more disulfide bonds to extra keratin, it can specifically catalyze more covalent crosslinking in keratin. TG enzyme can promote the crosslink not only intra and inter molecular chains of the broken hair fibers, but also between the extra keratin molecule chains and the molecular chains of the hair fibers, making the repair effect more significant and quasi-permanent [10-12].

In this study, we applied TG enzyme to repair the damaged hairs and obtain the modified protein fibers, and measured its repair effect. Overall, our study provides a theoretical basis for enzymatic hair care and a new idea for biological hair care. Furthermore it confirmed the great potential of application of various protein-based materials.

\section{MATERIALS AND METHODS}

\section{Preparation of keratin repair solution and} repair process

Keratin repair solution was prepared by diluting the mixture of $12 \mathrm{ml}$ of $5 \%(\mathrm{w} / \mathrm{v})$ hydrolyzed keratin solution of 20-30 KDa molecular weight and $8 \mathrm{ml}$ of $5 \%(\mathrm{w} / \mathrm{v})$ hydrolyzed keratin solution of $0.7-$ $1.4 \mathrm{KDa}$ molecular weight to $100 \mathrm{ml}$ with water. For hair repair, $1 \mathrm{~g}$ of damaged hair samples, $\sim 30 \mathrm{~cm}$ long, were soaked in the prepared keratin repair solution at $40^{\circ} \mathrm{C}$ for $90 \mathrm{~min}$. After wash with deionized water for 3-5 times, the repaired samples were dried naturally, labeled as KRS, and set aside for future uses.

\section{Preparation of TG enzyme repair solution and repair process}

TG enzyme repair solution was prepared by dissolving $0.1 \mathrm{~g}$ TG enzyme powder (activity $4700 \mathrm{U} / \mathrm{g}$ ) in $100 \mathrm{ml}$ deionized water. For hair repair, $1 \mathrm{~g}$ of damaged hair samples were soaked in the prepared TG enzyme repair solution at $40^{\circ} \mathrm{C}$ for $90 \mathrm{~min}$. After having been washed with deionized water for 35 times, the repaired samples were dried naturally, labeled as TGRS, and set aside for future uses.

\section{Preparation of keratin-TG enzyme combined repair solution and repair process}

After a series of screening and optimization experiments, preparation of keratin-TG enzyme combined repair solution and repair process were finalized as the following. First, $1 \mathrm{~g}$ of damaged hair samples 
were soaked in $100 \mathrm{ml}$ keratin repair solution at $40^{\circ} \mathrm{C}$ for $30 \mathrm{~min}$. After having been washed with deionized water for 3-5 times, the samples were soaked in TG enzyme repair solution at $40^{\circ} \mathrm{C}$ for $90 \mathrm{~min}$. At last, the samples were washed again with deionized water 3-5 times, dried naturally, labeled as TG-KRS and set aside for future uses.

\section{Characteristic determination of the repaired hair samples}

\section{Determination of alkali solubility}

A certain amount of hair samples with constant dry weight were treated with $100 \mathrm{ml}$ of $0.2 \mathrm{~mol} / 1$ sodium hydroxide solution at $65^{\circ} \mathrm{C}$ for $60 \mathrm{~min}$. These samples were filtered, washed with distilled water, neutralized with $10 \mathrm{ml}$ of diluted acetic acid, and dried at $65^{\circ} \mathrm{C}$ to constant weight. The alkali solubility, $a$, was calculated as

$$
a=\frac{X(1-G)-W}{X(1-G)} \times 100 \%,
$$

where $X$ is the original hair mass, $g$; $G$ is the \% moisture content (approximately equal to 0 ); and $W$ is the residual hair mass after alkali treatment, g.

\section{Determination of hair fracture stress and fracture strain}

The experiment was performed using an electronic fiber strength test machine following the FZ/T 01030-1993 standard. For each sample, 50 parallel hairs were randomly selected. After eliminating 10 maximum and 10 minimum values, the average value of the remaining 30 data was taken as the fracture stress and fracture strain of the hair sample.

\section{Determination of water absorption capability}

The hair samples were fixed on glass slides and dynamically observed under a $\mathrm{KH}-7700$ digital threedimensional video microscope (HIROX, Japan) before and after addition of water droplets on the surface by taking images every $30 \mathrm{~s}$ for a total of $30 \mathrm{~min}$. These images were then analyzed with Image-Pro Plus 6.0 software, as shown in Fig. 2, where the left images are taken before water absorption, and the right ones after water absorption. The diameter of hair sample on each image was measured three times and their average was regarded as the diameter of the sample at the moment. The hair swelling rate, $E$, was calculated as

$$
E=\frac{d_{1}-d_{0}}{d_{0}} \times 100 \%,
$$
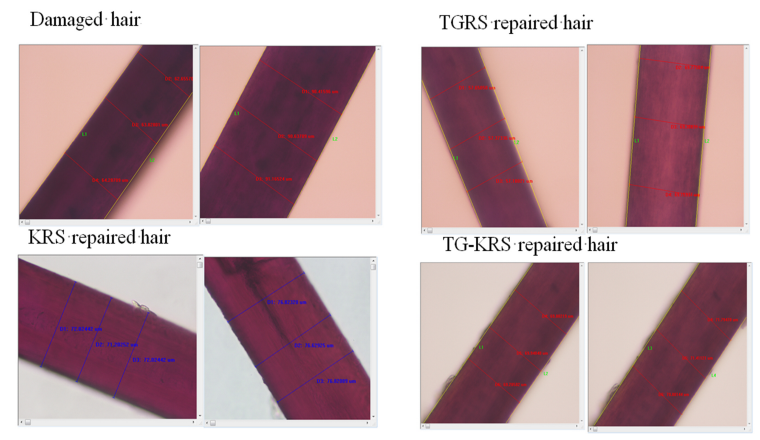

Fig. 2 Schematic diagrams of different hair samples before and after water absorption.

Table 1 Characteristics of damaged hair samples before and after repair using different methods.

\begin{tabular}{lccrr}
\hline & $\begin{array}{c}\text { Alkali } \\
\text { solubility }\end{array}$ & $\begin{array}{c}\text { Fracture } \\
\text { stress }\end{array}$ & $\begin{array}{c}\text { Fracture } \\
\text { strain }\end{array}$ & $\begin{array}{c}\text { Swelling } \\
\text { rate }\end{array}$ \\
\hline Damaged hair & $16.14 \%$ & $0.832 \mathrm{~N}$ & $8.81 \mathrm{~mm}$ & $38.34 \%$ \\
KRS & $8.19 \%$ & $1.545 \mathrm{~N}$ & $9.71 \mathrm{~mm}$ & $14.09 \%$ \\
TGRS & $6.99 \%$ & $1.042 \mathrm{~N}$ & $9.42 \mathrm{~mm}$ & $21.02 \%$ \\
TG-KRS & $4.92 \%$ & $1.668 \mathrm{~N}$ & $10.17 \mathrm{~mm}$ & $2.34 \%$ \\
\hline
\end{tabular}

KRS, keratin repair solution; TGRS, TG enzyme repair solution; TG-KRS, keratin-TG enzyme combined repair solution.

where $d_{1}$ is the diameter after water adsorption, and $d_{0}$ is the diameter before water adsorption.

\section{Structural characterization of hair samples}

The surface attenuation total reflection of the hair samples was measured with Nicolet10 Fourier Transform Infrared Spectrometer with wavelength in the range of $500-4000 \mathrm{~cm}^{-1}$. Changes in crystallization degree of hair samples were determined with X-ray diffractometer (Brook, Germany) in the scanning range of $5-60^{\circ}$ at the scanning rate of $2^{\circ} / \mathrm{min}$. The surface characters of samples were measured with Hitachi Regulus 8220 scanning electron microscope (SEM) at the voltage of $5.0 \mathrm{kV}$. The microscopic topography of the sample surface was scanned with a MultiMode8 Atomic force microscope (Brook, Germany).

\section{RESULTS AND DISCUSSION}

\section{Characteristics of damaged hair samples before} and after repair

Table 1 shows the characteristics of the damaged hair samples before and after different repair methods. All three repairing methods had certain repair effects. Among them, keratin-TG enzyme combined 
repair solution had the best repair effect with alkali solubility reduced from $16.14 \%$ to $4.92 \%$, the fracture stress increased from $0.832 \mathrm{~N}$ to $1.668 \mathrm{~N}$, the fracture strain increased from $8.81 \mathrm{~mm}$ to $10.17 \mathrm{~mm}$, and swelling rate reduced from $38.34 \%$ to $2.34 \%$. Hair repair with TG enzyme repair solution mainly depends on the catalytic action of TG enzyme to crosslink intra- or inter-molecular chains in the hair fibers [13]. Hair repair with keratin repair solution mainly depends on penetration of keratin into the hair cortex, where it interacts with and crosslink to the exposed hydrophilic groups in the damaged hair to form covalent bonds [14]. While in hair repair with TG enzyme repair solution, the catalytic effect of TG enzyme promotes crosslinks not only intra- and inter- of molecular chains in the damaged hairs, but also between the extra keratin molecules and the fiber molecular chains in the hair, so that more keratin and broken fiber molecular chains participate in the cross-linking reaction, which, to a greater extent, improves the mechanical properties of the damaged hair and reduces the alkali solubility [13-16].

\section{Structural characterization of damaged hairs before and after repair}

Fig. 3a shows the infrared spectra of damaged hair as well as KRS, TGRS and TG-KRS, where the characteristic peaks at $3100 \mathrm{~cm}^{-1}, 1689 \mathrm{~cm}^{-1}$, $1531 \mathrm{~cm}^{-1}$, and $1290 \mathrm{~cm}^{-1}$ correspond to the amide group (-CONH-), amide I band, amide II band, and amide III band, respectively.

Compared to the non-repaired hair samples, all treatments did not shift the peak positions of the hair samples, but significantly increased the area of each peak and the abundance of the amide bond, with keratin-TG enzyme combined repair solution treatment showing the most significant effect. These results indicate that TG enzyme treatment does not affect the fine structure of the hair proteins while improving the physicochemical properties of the damaged hair. The increase in the abundance of amide bonds indicates that addition of keratin provides more repair sites for TG enzyme to form a dense spatial network structure via crosslinks and increase in hair strength and stress resistance [17-19].

Fig. 3b shows the X-ray diffraction patterns of damaged hair as well as KRS, TGRS, and TGKRS. The higher the crystallinity is, the greater the percentage of the crystalline region in the entire fiber [20]. Because of the dense structure and high density of the crystalline zone, there are more strong
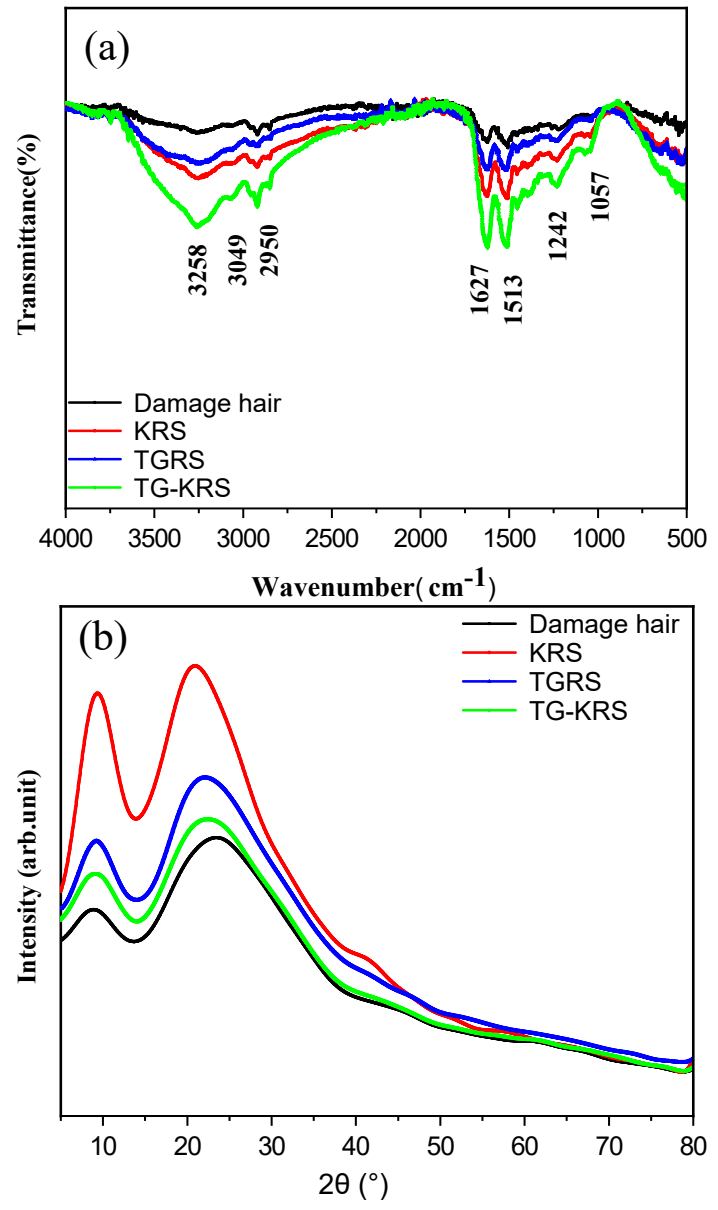

Fig. 3 (a) ATR-FTIR diagram and (b) XRD diagram of the damaged hair sample as well as KRS, TGRS and TG-KRS.

chemical bonds between the molecules, which results in improved tensile strength, toughness and stability of the fiber. Therefore, water molecules and chemicals are not easy to enter, which also leads to a decrease in alkali solubility. The crystallinity of KRS is closest to that of the damaged hair samples; while those of TGRS and TG-KRS were $16 \%$ and $35 \%$ higher than that of the damaged hair samples, respectively. These results show that during the repair process, TG enzyme and keratin molecules could easily penetrate into the random regions of the hair fibers, so that crosslink reaction reoccurs both inter- and intra- molecular chains under the catalytic action of TG enzyme. In addition, keratin also reacts with the amino acid residues inside the hair fibers. Thus, the random regions inside the protein fiber under the action of chemical bonds affect the spatial arrangement of the original molecular chains, which improves the regularity of the 


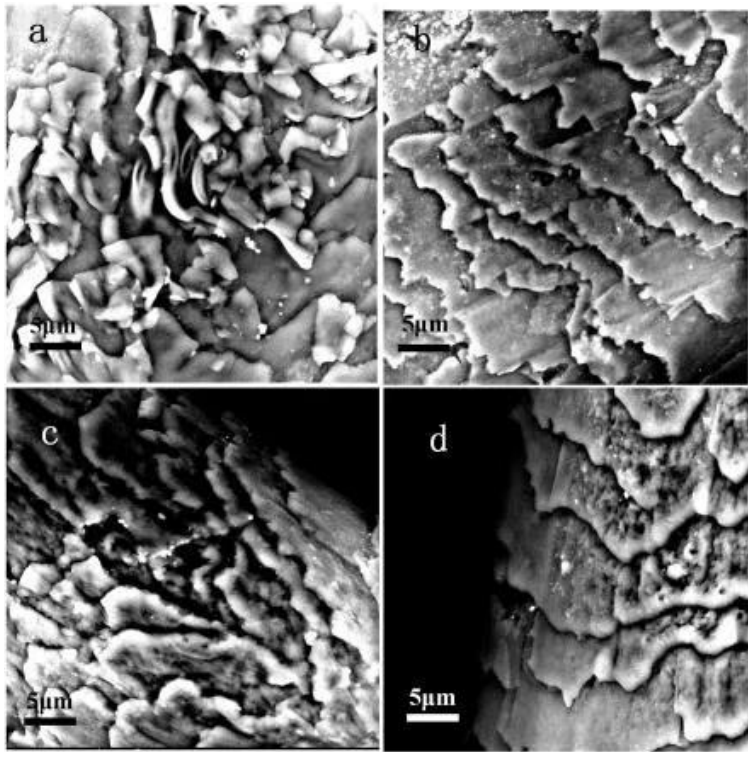

Fig. 4 SEM images of (a) damaged hair samples, (b) KRS, (c) TGRS and (d) TG-KRS.

molecular chains, leading to increased crystallization degree of the repaired hair samples [20-23].

Fig. 4 shows the scanning electron micrographs of the damaged hair sample as well as KRS, TGRS and TG-KRS. The curled, warped and deformed scales due to external factors were clearly seen in the image of the damaged hair sample (Fig. 4a). White attachments on the slightly rough surface of the hairline were seen in the image of KRS (Fig. 4b) possibly due to keratin attachment. Closed and slightly warped scales were seen in the image of TGRS (Fig. 4c). A small amount of attachments and well-closed, un-wrapped scales were seen in the image of TG-KRS. The attachments under keratin treatment alone only adhere to the hair surface for a very short period and are easy to fall off to form "dandruff". In addition, TG enzyme treatment alone is unable to completely repair the warped scales, indicating that the crosslink sites provided by TG enzyme are insufficient to completely repair hair damages. For repair with combined TG enzyme and keratin, keratin molecules that are not involved in the hair repair will react with TG enzyme to form a flocculent precipitate, which is easy to wash away; while the keratin involved in the repair are crosslinked to the hair protein fibers, or other keratin molecules on the protein fiber, so that the treated hair protein fiber not only has a smoother surface, but also stronger mechanical properties [24, 25].

Fig. 5 shows the topology of the hair samples
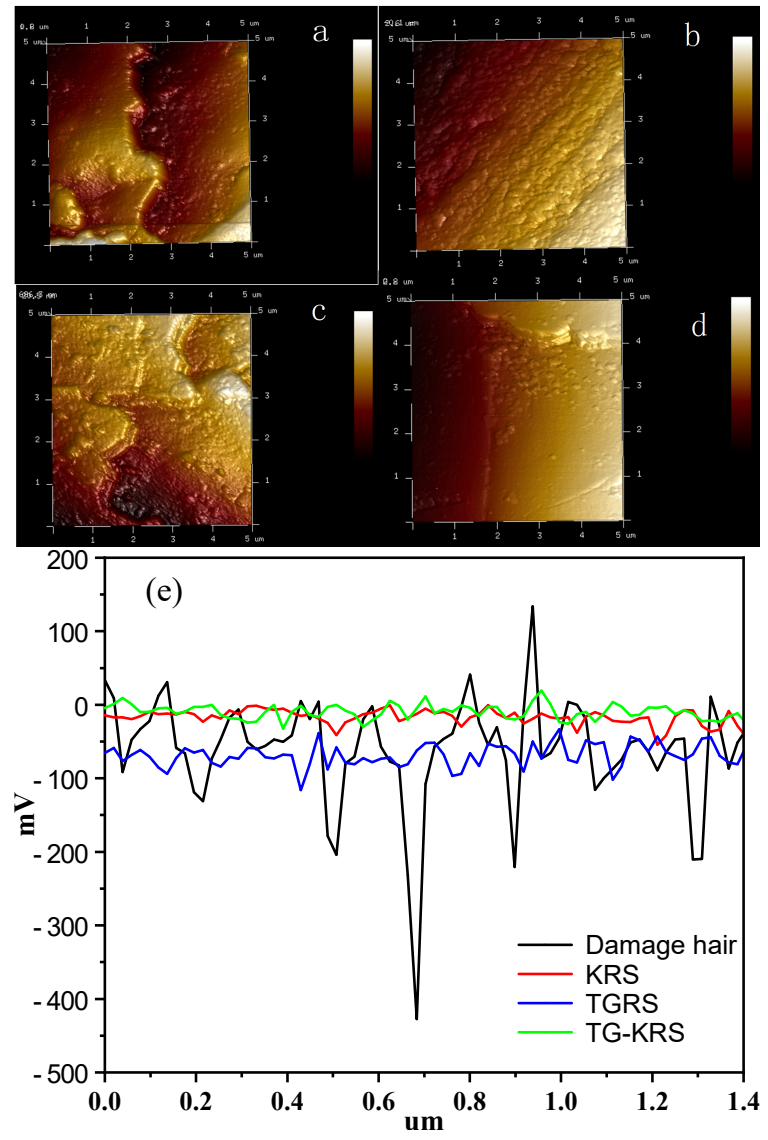

Fig. 5 AFM images of (a) damaged hair, (b) KRS, (c) TGRS and (d) TG-KRS, and (e) the calculated surface roughness.

under AFM. The surface scales of the damaged hair samples were destroyed, showing increased surface roughness (Fig. 5a). This is mainly because the hair scales are turned upward, resulting in a larger gap between the hair scales and the hair shaft, which enlarges the measurement result. The surface scales of KRS or TGRS (Fig. 5b-c) are only slightly closed than those of the damaged hair samples. Especially, there are many attachments on the surface of KRS. Due to the film-forming properties of keratin, a thin film will be formed on the hair surface with obvious graininess. The scales of TG-KRS are closed with regular surface structure, smooth surface and fewer foreign objects (Fig. 5d). These results are consistent with the infrared and SEM results, and prove that TG enzyme plays an important role in the repair of the damaged hairs.

Fig. 5e shows the roughness of the hair surface analyzed through NanoScope analysis 1.5 and origin 2018 software. The surface roughness of 
the damaged hair samples is extremely high. TG enzyme repair solution treatment only slightly improves the surface roughness of the damaged hair samples; while keratin repair solution obviously improves the surface roughness and makes the surface become smooth. More importantly, treatment with keratin-TG enzyme combined repair solution significantly improves the surface roughness and makes the surface even smoother compared with treatment with keratin repair solution, which are consistent with the results observed by SEM. Overall, together with infrared and XRD analyses, the results indicate that treatment with keratin-TG enzyme combined repair solution leads to crosslinks of a large amount of keratin or keratin peptides to the damaged sites of the hair under the action of TG enzyme or via disulfide bond recombination occurring between the proteins, so that the surface of the damaged hair is modified by keratin and TG enzyme and becomes smooth [26].

\section{CONCLUSION}

In this study, we obtained a keratin repair solution with the best molecular weight ratio and used TG enzyme as additive for double repair via protein recombination and TG enzyme crosslink to obtain modified protein fibers with better quality. Compared with keratin repair alone and TG enzyme repair alone, the use of keratin-TG enzyme combined repair solution to repair hair samples not only significantly reduces the alkali solubility and water swelling rate of the hair, but also causes the breaking stress of the hair and the fracture strain is significantly increased. This method not only ameliorates the quality of artificially and naturally damaged hairs, improves damage resistance, increases gloss and toughness of human hairs, and prevents dry and frizzy hair texture, but also opens up a new direction of green, environmentally friendly and efficient biological care, thus providing great potentials for the application of various protein-based materials.

Acknowledgements: The authors are grateful for the financial support of the Major Scientific and Technological Innovation Project in Shandong Province (Grant No. 2019JZZY011001).

\section{REFERENCES}

1. Tonin C, Aluigi A, Songia MB, D`Arrigo C, Mormino M, Vineis C (2004) Thermoanalytical characterisation of modified keratin fibres. $J$ Therm Anal Calorim 77, 987-996.
2. Watt IC, Morris R (1969) Factors affecting the thermal contraction of keratin fibres. J Polym Sci Pol 229, 29-33.

3. Barba C, Scott S, Roddick LA, Kelly R, Manich AM, Parra JL (2010) Restoring important hair properties with wool keratin proteins and peptides. Fiber Polym 11, 1055-1061.

4. Barba CM, Martí Roddick-LA, Manich A, Carilla J, Parra JL, Coderch L (2010) Effect of wool keratin proteins and peptides on hair water sorption kinetics. $J$ Therm Anal Calorim 102, 43-48.

5. Yang X, Lin ZB, Cui SX (2007) Effect of concentration of wool keratin on the rebuilding of disulfur bond. Chinese Sci Bull 20, 142-145.

6. Fan J, Yu WD (2012) High yield preparation of keratin powder from wool fiber. Fiber Polym 13, 1044-1049.

7. Montazer M, Pakdel E, Moghadam MB (2010) Nano titanium dioxide on wool keratin as UV absorber stabilized by butane tetra carboxylic acid (btca): a statistical prospect. Fiber Polym 11, 967-975.

8. Drack M, Wimmer R (2007) Woolrock: a material for technical use consisting of keratin. J Mater Sci 42, 6183-6187.

9. Ragot J (1969) Influence de certains agents de sterilisation sur la keratine. Mycopathol Mycol Appl 39, 177-186.

10. Mathison GE (1966) An autoradiographic technique for the detection of cystine and peptides containing cystine or its derivatives in keratin digest. Mycopathol Mycol Appl 27, 225-232.

11. Aluigi A, Tonetti C, Rombaldoni F, Puglia D, Fortunati E, Armentano I, Santulli C, Torre L, et al (2014) Keratins extracted from merino wool and brown alpaca fibres as potential fillers for plla-based biocomposites. J Mater Sci 49, 6257-6269.

12. Reddy N, Yang Y (2007) Structure and properties of chicken feather barbs as natural protein fibers. $J$ Polym Environ 15, 81-87.

13. Defu S, Xiangyang L, Qiulin W, Xingcai C (2010) Study on enhancing the gel strength of shark fin pill using glutamine transaminase and gellan gum. Acad Period Farm Prod Process 1, 1-3.

14. Wojciechowska E, Włochowicz A, WesełuchaBirczyńska A (1999) Application of fouriertransform infrared and raman spectroscopy to study degradation of the wool fiber keratin. J Mol Struct 511, 307-318.

15. Crighton JS, Findon WM (1977) A thermoanalytical study of treated wools. $J$ Therm Anal 11, 305-321.

16. Tonin C, Aluigi A, Vineis C, Varesano A, Montarsolo A, Ferrero F (2007) Thermal and structural characterization of poly(ethylene-oxide)/keratin blend films. $J$ Therm Anal Calorim 89, 601-608.

17. Chun-Li D, Guo-Quan Z (2013) Effects of transglutaminase treatment on the quality of buckwheat protein. Food Res Dev 34, 25-29. 
18. Molina R, Jovančić P, Comelles F, Bertran E, Erra P (2012) Shrink-resistance and wetting properties of keratin fibres treated by glow discharge. $J$ Adhes Sci Technol 16, 1469-1485.

19. Kovarskii AL, Kasparov VV, Krivandin AV (2017) Epr spectroscopic and x-ray diffraction studies of carbon fibers with different mechanical properties. Russ $J$ Phys Chem B 11, 233-241.

20. Tatiana N, Vladimir K, Mikhail I (2019) Sorption of d-metal cations by keratin from aqueous solutions. $J$ Environ Chem Eng 7, ID 103417.

21. Woods EF (1989) Aggregation of wool keratin intermediate filament proteins [J]. Int J Biol Macromol 11, 278-284.

22. Fernández-d'Arlas B (2019) Tough and functional cross-linked bioplastics from sheep wool keratin. Sci Rep 9, 1-12.
23. Giuri D, Barbalinardo M, Sotgiu G, Zamboni R, Nocchetti M, Donnadio A, Corticelli F, Valle F, et al (2019) Nano-hybrid electrospun non-woven mats made of wool keratin and hydrotalcites as potential bio-active wound dressings. Nanoscale 13, 6422-6430.

24. Skertchly ARB (1964) 30 - A unified hypothesis for the physical structure and deformation behavior of wool keratin. $J$ Text Trans 55, 324-334.

25. Jeffrey EP, Duane PH, Sivasangary G, Joy LW, Bede VS, Santanu DC, Ancy T, Stefan C, et al (2015) The proteomics of wool fibre morphogenesis. J Struct Biol 191, 341-351.

26. Dowling LM, Crewther WG, Inglis AS (1986) The primary structure of component $8 \mathrm{c}-1$, a subunit protein of intermediate filaments in wool keratin: relationships with proteins from other intermediate filaments. Biochem J 236, 695-703. 